\title{
The Impact of Perceived Hapkido Service Quality on Exercise Continuation and Recommendation Intentions, with a Focus on Korean Middle and High School Students
}

\author{
Tae-Seung Park ${ }^{1}$, Jun-Su Kim ${ }^{2, *,+}$ and Jiyoun Kim ${ }^{3, *,+}$ \\ 1 Department of Taekwondo Education Convergence, Shinhan University, Uijeongbu-si 11644, Korea; \\ pts81@naver.com \\ 2 Department of Sports \& Outdoors, College of Health Industry, Eulji University, Seongnam-si 13735, Korea \\ 3 Department of Exercise Rehabilitation \& Welfare, Gachon University, Incheon 21936, Korea \\ * Correspondence: kjskjs77777@gmail.com (J.-S.K.); eve14jiyoun@gachon.ac.kr (J.K.); \\ Tel.: +82-10-3764-7005 (J.-S.K.); +82-10-9788-6839 (J.K.) \\ + These two authors contributed equally to this work.
}

check for updates

Citation: Park, T.-S.; Kim, J.-S.; Kim, J. The Impact of Perceived Hapkido Service Quality on Exercise Continuation and Recommendation Intentions, with a Focus on Korean Middle and High School Students. Sustainability 2021, 13, 3389. https://doi.org/10.3390/ su13063389

Academic Editors: Adilson Marques and Miguel Peralta

Received: 27 February 2021

Accepted: 15 March 2021

Published: 18 March 2021

Publisher's Note: MDPI stays neutral with regard to jurisdictional claims in published maps and institutional affiliations.

Copyright: (c) 2021 by the authors. Licensee MDPI, Basel, Switzerland. This article is an open access article distributed under the terms and conditions of the Creative Commons Attribution (CC BY) license (https:// creativecommons.org/licenses/by/ $4.0 /)$.

\begin{abstract}
This research analyzes the impact of quality of service as perceived by Hapkido students on their exercise continuation and recommendation intentions. It also identifies measures to reduce the rate of student dropout, strengthen competitiveness, and create more efficient marketing strategies for consumer patterns that are rapidly diversifying Hapkido. A questionnaire survey method was conducted with 300 middle and high school students, aged 14-19 years, with between three months and two years of Hapkido training in Incheon and Bucheon during March and April 2019. Frequency, factor, reliability, correlation, and standard multiple regression analyses were conducted on the surveyed data. The study concludes that, first, considering the impact of service quality on exercise continuation intention, service quality positively affects reliability, personification, and perceptual openness; in terms of possibility, it positively affects typicality, personification, and perceptual openness; and in terms of reinforcement, it positively affects reliability and perceptual openness. Second, regarding the impact of service quality on recommendation intention, it positively affects reliability, personification, and perceptual openness. Third, exercise continuation intention positively affects recommendation intention. This study suggests that various efforts should be made to increase the reliability, tangibility, confidence, and empathy of the service quality to increase the exercise continuation and recommendation intentions of the trainees of Hapkido gymnasiums.
\end{abstract}

Keywords: Hapkido; service quality; quality on exercise continuation; recommendation intentions

\section{Introduction}

In Hapkido, a traditional Korean martial art, nature is considered the source of energy in human beings. Hapkido utilizes this energy to train the mind and body by unifying them. Since 1948, in South Korea, 6004 Hapkido divisions have been registered with the Korea Sports Council; moreover, the knowledge of Hapkido has been recognized with additional points on civil service tests by government agencies such as the National Police Agency and National Intelligence Service [1,2]. This indicates the value of Hapkido, which has been adopted as an essential martial art by national institutions with a positive influence on youths' health, confidence, and leadership [3]. Hapkido training relaxes tension and helps maintain mental and physical health. Moreover, it fosters a sense of fairness, duty, accomplishment, psychological stability, patience, self-control, willpower, self-esteem, and justice. It can also be effective for personality development, as it can cultivate closeness, friendship, mutual respect, and concession through the confrontation with a partner [4]. Sports such as Hapkido provide important motivation for students who wish to pursue a career in national security, as well as improving the youth's education and physical 
ability $[5,6]$. However, despite the positive effects of adolescent personality education and the physical improvement required in modern society, the number of Hapkido gyms is decreasing, similar to other after-school programs, because of a decrease in birth rate in Korea and a drop in private education spending due to the economic recession [7]. This has caused overwhelming competition, such as giving away expensive gifts when the new students register, and it prevents Hapkido from performing its original purpose, which is nurturing and developing one's character [8].

This marketing strategy can be effective in attracting new students and maintaining them for a short period of time; however, it is difficult to achieve the students' training goals without establishing a true long-term relationship. To continuously develop Hapkido, identifying the consumers' rapidly changing needs to secure a variety of students of all ages, regardless of age or gender, is important. In addition, efforts are needed to improve the quality of services, including providing high-quality educational programs, managing facilities at the Hapkido gymnasiums to fulfill the needs of students and the parents' desire to foster their children's physical health and a positive mind, and developing the abilities of coaches and leaders.

Service quality is the quality perceived by the consumer as a comprehensive impression of the tangible and intangible services provided to them. In general, service quality is rated as friendly or superior when it meets or exceeds customer expectations, respectively [9]. This concept comprises psychological studies [10]. The higher the quality of service that is recognized, the higher the intention of re-registration and of recommendation to others [11]. In other words, Hapkido students who maintain mutual trust through a high quality of service not only have higher intentions to continue training but also recommend registering for Hapkido to friends and acquaintances [12].

Despite the research on service quality continuing as part of sports marketing strategies in various martial arts and sports events in Korea $[13,14]$, in the case of Hapkido, prior research is insufficient.

Therefore, this study considers the characteristics of Hapkido in South Korea. Specifically, it analyzes the impact of the service quality that is perceived by a particular age group of Hapkido students on exercise continuity and recommendation intentions. This research aims to provide basic data for the operation of Hapkido gymnasiums in South Korea, and improve service quality.

\section{Research Method}

This study employs the following research procedures, tools, and data processing methods.

\subsection{Research Subjects}

This research was conducted with the approval of the Research Ethics Committee to study the effect of the service quality as perceived by Hapkido trainees on their exercise continuation and recommendation intentions (INHA-IRB-20191029012). The subjects were 300 Hapkido students aged 14-19 years who had trained from three months to two years in Incheon and Bucheon. The survey duration was one month, from March to April 2019.

The subjects were middle and high school students selected using the convenience sampling method. The researchers explained the study's purpose and survey contents to the director of Hapkido, who trained these students. After obtaining consent, a total of 300 questionnaires were distributed to the participants. The questionnaires were collected directly by the investigator immediately after they were filled by the subjects using a self-administration method. Eleven questionnaires were excluded because they were not answered by the students, so 289 were finally used and analyzed. The responses were assessed through data processing and statistical analysis. The demographic characteristics of the participants are shown in Table 1. 
Table 1. Demographic characteristics.

\begin{tabular}{cccc}
\hline & Characteristics & Frequency (Persons) & Ratio (\%) \\
\hline & 1st grade in middle school & 36 & 12.5 \\
2nd grade in middle school & 41 & 14.2 \\
School year & 3rd grade in junior high school & 35 & 12.1 \\
& 1st grade in high school & 52 & 18.0 \\
& 2nd grade in high school & 68 & 23.5 \\
& Senior in high school & 57 & 19.7 \\
\hline \multirow{3}{*}{$\begin{array}{c}\text { Participation period } \\
\text { (5 practices a week) }\end{array}$} & More than 3 months & 17 & 5.9 \\
& 3 6 months & 42 & 14.5 \\
& 6 months 1 year & 100 & 34.6 \\
Degree of participation & 1 2 years & 50 & 17.3 \\
(times per week) & Less than 2 years & 80 & 27.7 \\
& Less than 3 times & 34 & 11.8 \\
& 4 times & 87 & 30.1 \\
& More than 5 times & 168 & 58.1 \\
\hline \multirow{2}{*}{ Recommendation } & Friends' recommendation & 56 & 19.4 \\
& Self-motivation & 102 & 35.3 \\
& Parents' recommendation & 73 & 25.3 \\
& Siblings' recommendation & 27 & 9.3 \\
& Others (e.g., promotional & 31 & 10.7 \\
\hline & materials and marketing) & 289 & $100 \%$ \\
\hline
\end{tabular}

\subsection{Investigation Tool}

A questionnaire was used as the survey tool for this study. All items in the questionnaire were organized according to the study purpose based on previous studies and theories. In addition, the questionnaire was reorganized by conducting a group meeting of experts, after drafting based on various preceding research data to understand the effects of service quality as perceived by Hapkido students on their exercise continuation and recommendation intentions.

\subsubsection{Service Quality}

To measure the service quality factor in this study, the items used by Hwang [15], which were developed based on the five factors of Parasuramam et al. [16], were used after revising and supplementing them to suit this study's purpose. A total of 23 questions were used for the following subfactors of service quality: tangibility, which is the appearance of physical facilities and equipment (4 questions); reliability, the ability to perform the promised service accurately (5 questions); responsiveness, which is the willingness to help customers and provide prompt service (4 questions); confidence, which is the knowledge and courtesy shown by the employees (5 questions); and empathy, which is the caring, individualized attention the firm provides to its customers (5 questions). Each was measured on a five-point Likert scale.

\subsubsection{Intention to Continue Exercise}

The test tool to measure exercise continuation intention was the Exercise Adherence Questionnaire developed by Corbin and Lindsey [17]. The questions were taken from Lee et al. [18]; they were translated, modified, and supplemented according to this study's purpose.

A total of 14 questions for the following subfactors of persistence intention were used: possibility, which is the external motivational factors that help when trying to perform exercise planning (5 questions); tendency, which comprises the internal motivators that help make exercise a part of everyday life (5 questions); and reinforcement, which comprises the external motivation factors, such as friends, co-workers, coaches, and professionals, who 
help you continue to exercise regularly (4 questions). Each was measured on a five-point Likert scale.

\subsubsection{Recommendation Intention}

Based on the previous study by Kim et al. [19], to measure the reasons for recommending Hapkido, Choi's [20] recommendation intention scale was used. The scale was modified and supplemented to suit this study. Recommendation intention was measured using four questions using a five-point Likert scale.

\subsection{Validity and Reliability}

The feasibility verification methods adopted in this study were as follows. First, the questionnaire was developed based on the literature and expert opinion gathering, expert meetings, and preliminary inspections. Second, the completed draft questionnaire was reviewed by a group of experts who had doctorates or were professors in the sports management industry. Third, exploratory factor analysis was conducted to verify the questionnaire's validity.

\subsubsection{Validity Verification}

Exploratory factors were used to verify the validity of the analysis. Maximum likelihood was used for factor extraction, and the exploratory factor analysis used principal component analysis and varimax rotation with a right-angle rotation method.

\section{Service Quality}

Service quality was composed of 23 measurement variables based on previous studies. Principal component analysis was used for the extraction model, and the varimax method, a perpendicular rotation method that assumes independence between factors, was used. After specifying factor loadings of at least 0.5 and repeatedly performing factor analysis while considering commonality and the researcher's intention, five factors with eigenvalues of 1 or more for the 23 measurement variables were extracted. The Kaiser-Meyer-Olkin (KMO) coefficient for service quality was 0.888 , which can be considered appropriate for factor analysis. Bartlett's test of sphericity showed a value of 2210.222, and $p<0.001$. The explanatory power for the total variance of these factors was $55.746 \%$. Table 2 presents the results.

Table 2. Service quality factor analysis results.

\begin{tabular}{|c|c|c|c|c|c|}
\hline \multirow{2}{*}{ Measurement Item } & \multicolumn{5}{|c|}{ Composition } \\
\hline & Empathy & Responsiveness & Reliability & Tangible & Confidence \\
\hline Empathy5 & 0.761 & 0.157 & 0.176 & 0.038 & 0.085 \\
\hline Empathy1 & 0.667 & 0.394 & 0.017 & -0.054 & 0.127 \\
\hline Empathy3 & 0.786 & 0.137 & 0.379 & 0.015 & 0.078 \\
\hline Empathy4 & 0.651 & 0.317 & 0.080 & 0.403 & -0.034 \\
\hline Empathy2 & 0.612 & 0.385 & 0.291 & 0.014 & 0.037 \\
\hline Responsiveness2 & 0.150 & 0.719 & 0.289 & -0.013 & 0.118 \\
\hline Responsiveness4 & -0.043 & 0.677 & 0.364 & 0.103 & 0.286 \\
\hline Responsiveness1 & 0.237 & 0.672 & 0.273 & -0.083 & 0.297 \\
\hline Responsiveness3 & 0.264 & 0.624 & 0.318 & 0.264 & 0.107 \\
\hline Reliability2 & 0.233 & -0.110 & 0.660 & 0.346 & 0.047 \\
\hline Reliability4 & 0.311 & 0.119 & 0.640 & 0.181 & 0.057 \\
\hline Reliability5 & 0.312 & 0.014 & 0.622 & -0.025 & 0.201 \\
\hline Reliability3 & 0.259 & 0.082 & 0.612 & -0.004 & 0.041 \\
\hline Reliability1 & 0.378 & 0.047 & 0.702 & 0.290 & 0.139 \\
\hline Tangible4 & -0.083 & 0.385 & 0.057 & 0.684 & 0.111 \\
\hline Tangible3 & 0.209 & 0.193 & 0.167 & 0.625 & 0.113 \\
\hline Tangible2 & 0.304 & 0.328 & -0.077 & 0.759 & 0.077 \\
\hline Tangible1 & 0.249 & 0.266 & 0.222 & 0.701 & -0.043 \\
\hline Confidence5 & 0.094 & 0.364 & 0.184 & 0.200 & 0.694 \\
\hline Confidence1 & -0.066 & -0.009 & 0.000 & 0.363 & 0.677 \\
\hline
\end{tabular}


Table 2. Cont.

\begin{tabular}{cccccc}
\hline \multirow{2}{*}{ Measurement Item } & \multicolumn{5}{c}{ Composition } \\
\cline { 2 - 6 } & Empathy & Responsiveness & Reliability & Tangible & Confidence \\
\hline Confidence3 & 0.171 & 0.099 & -0.036 & 0.288 & 0.646 \\
Confidence 2 & 0.207 & 0.049 & 0.274 & 0.230 & 0.729 \\
Confidence4 & -0.125 & 0.262 & 0.111 & 0.201 & 0.669 \\
\hline Eigenvalue & 3.145 & 2.732 & 2.498 & 2.261 & 2.185 \\
\hline Total sample variance ratio & 13.676 & 11.876 & 10.863 & 9.831 & 9.500 \\
\hline Kaiser-Meyer-Olkin coefficient & \multicolumn{5}{c}{0.888} \\
\hline Bartlett's test & \multicolumn{5}{c}{0.001} \\
\hline Significance probability & \multicolumn{5}{c}{}
\end{tabular}

Intention to Continue Exercising

Exercise continuation intention was composed of 14 measurement variables based on previous studies. Principal component analysis was used as the extraction model, and the varimax method was also used. The factor analysis was performed repeatedly in consideration of the commonality and intention of the researcher, with a factor loading value of 0.5 , and three factors with eigenvalues of 1 or more for 14 measurement items were extracted. The KMO coefficient for the exercise continuation intention was 0.877 , which can be considered appropriate for the factor analysis. Bartlett's test of sphericity showed a value of 1301.190 , and $p<0.001$. The explanatory power for the total variance of these factors was $57.708 \%$. Table 3 presents the results.

Table 3. Exercise continuity factor analysis results.

\begin{tabular}{cccc}
\hline \multirow{2}{*}{ Measurement Item } & \multicolumn{3}{c}{ Composition } \\
\cline { 2 - 4 } & Tendency & Possibility & Reinforcement \\
\hline Tendency4 & 0.611 & 0.347 & 0.024 \\
Tendency5 & 0.563 & 0.367 & 0.167 \\
Tendency3 & 0.548 & 0.232 & 0.497 \\
Tendency2 & 0.792 & 0.079 & 0.192 \\
Tendency1 & 0.784 & 0.162 & -0.001 \\
\hline Possibility5 & 0.297 & 0.532 & 0.026 \\
Possibility3 & 0.257 & 0.725 & 0.165 \\
Possibility2 & -0.184 & 0.716 & 0.391 \\
Possibility4 & 0.245 & 0.624 & -0.075 \\
Possibility1 & 0.386 & 0.594 & 0.195 \\
\hline Reinforcement1 & 0.086 & 0.106 & 0.789 \\
Reinforcement2 & 0.105 & 0.165 & 0.692 \\
Reinforcement3 & 0.040 & 0.261 & 0.665 \\
Reinforcement4 & 0.379 & 0.249 & 0.578 \\
\hline Eigenvalue & 3.090 & 2.575 & 2.413 \\
\hline Total sample variance ratio & 22.075 & 18.396 & 17.237 \\
\hline Kaiser-Meyer-Olkin coefficient & & 0.877 & \\
\hline Bartlett's test & & Chi-square $=1301.190, \mathrm{df}=91$ \\
\hline Significance probability & & 0.001 & \\
\hline
\end{tabular}

\subsubsection{Reliability Verification}

Cronbach's $\alpha$ value, a measure of internal consistency, was used to analyze reliability for each measurement item. The result of all reliability analyses was 0.7 , so it was judged that there was no problem with the measurement tool's reliability [21]. Table 4 presents these results. 
Table 4. Reliability analysis results.

\begin{tabular}{|c|c|c|c|}
\hline \multicolumn{2}{|c|}{ Concept of Measurement } & \multirow{2}{*}{$\begin{array}{c}\text { Number of Questions } \\
5\end{array}$} & \multirow{2}{*}{$\begin{array}{c}\text { Cronbach's } \alpha \\
0.709\end{array}$} \\
\hline \multirow{5}{*}{ Service quality } & Empathy & & \\
\hline & Responsiveness & 4 & 0.708 \\
\hline & Reliability & 5 & 0.733 \\
\hline & Tangible & 4 & 0.704 \\
\hline & Confidence & 5 & 0.706 \\
\hline \multirow{3}{*}{ Sustainability } & Tendency & 5 & 0.724 \\
\hline & Possibility & 5 & 0.727 \\
\hline & Reinforcement & 4 & 0.736 \\
\hline \multicolumn{2}{|c|}{ Degree of recommendation } & 4 & 0.709 \\
\hline
\end{tabular}

\subsection{Data Processing}

The computerized data processing method was employed in this study using IBM SPSS 22.0. Frequency analysis was performed for the demographic analysis, and correlation analysis and regression were performed on service quality, exercise continuation intention, and recommendation intention. In addition, Cronbach's $\alpha$ was used for the exploratory factor and reliability analyses to confirm the survey tool's validity. The significance level of all statistical analyses was set at $p<0.05$.

\section{Research Results}

3.1. Correlation between Service Quality, Exercise Continuation Intention, and Recommendation Intention

All the above variables and subfactors showed a positive correlation in Table 5. Specifically, the correlation between each factor was significant: it was higher than 0.2 and lower than 0.8 , which is the criterion for multicollinearity.

Table 5. Results of the correlation analysis.

\begin{tabular}{|c|c|c|c|c|c|c|c|c|c|}
\hline & Empathy & Responsiveness & Reliability & Tangible & Confidence & Tendency & Possibility & Reinforcement & $\begin{array}{l}\text { Recommendation } \\
\text { Intention }\end{array}$ \\
\hline Empathy & 1 & & & & & & & & \\
\hline Responsiveness & $0.703^{* *}$ & 1 & & & & & & & \\
\hline Reliability & $0.790 * *$ & $0.554^{* *}$ & 1 & & & & & & \\
\hline Tangibility & $0.601 * *$ & $0.432 * *$ & & 1 & & & & & \\
\hline Confidence & $0.664^{* *}$ & $0.338^{* *}$ & $0.674^{* *}$ & $0.308^{* *}$ & 1 & & & & \\
\hline Tendency & $0.336^{* *}$ & $0.674^{* *}$ & $0.774^{* *}$ & $0.575^{* *}$ & $0.624^{* *}$ & 1 & & & \\
\hline Possibility & $0.532 * *$ & $0.553^{* *}$ & $0.546^{* *}$ & $0.570 * *$ & $0.474^{* *}$ & $0.443^{* *}$ & 1 & & \\
\hline Reinforcement & $0.443^{* *}$ & $0.452^{* *}$ & $0.439 * *$ & $0.664^{* *}$ & $0.665^{* *}$ & $0.501 * *$ & $0.409 * *$ & 1 & \\
\hline $\begin{array}{l}\text { Recommendation } \\
\text { intention }\end{array}$ & $0.504^{* *}$ & $0.460 * *$ & $0.758^{* *}$ & $0.309 * *$ & $0.590 * *$ & $0.677^{* *}$ & $0.346^{* *}$ & $0.663^{* *}$ & 1 \\
\hline
\end{tabular}

\subsection{Effect of Service Quality on the Exercise Continuation Intention}

The results of verifying the effect of service quality on exercise continuation intention's subfactors of tendency, possibility, and reinforcement were verified by multiple regression analysis and are shown in Table 6.

Regarding the effect of service quality on exercise continuation intention, tendency, possibility, and reinforcement all had statistically significant effects at a significance level of 0.001 . In the regression model of exercise continuation intention, the F-value was 39.849. $R^{2}=0.413$ for the regression equation indicated that it had $41.3 \%$ explanatory power, and reliability, confidence, and empathy were found to significantly affect exercise continuation intention.

The regression model of the possibility of exercise continuation intention showed a value of 40.303 . $R^{2}=0.416$ for the regression equation indicated that it had $41.6 \%$ explanatory power, and tangibility, confidence, and empathy had significantly affected possibility. 
Finally, the regression model of the reinforcement of the exercise continuation intention showed a value of 30.587. $R^{2}=0.351$ indicated that the regression equation showed $35.1 \%$ explanatory power, and confidence and empathy significantly affected reinforcement.

Table 6. Impact of service quality on exercise continuation intention.

\begin{tabular}{|c|c|c|c|c|c|c|c|c|c|c|c|c|c|}
\hline & & \multicolumn{4}{|c|}{ Tendency } & \multicolumn{4}{|c|}{ Possibility } & \multicolumn{4}{|c|}{ Reinforcement } \\
\hline & & B & SE & $\beta$ & $t$ & B & SE & $\beta$ & $t$ & B & SE & $\beta$ & $t$ \\
\hline \multicolumn{2}{|c|}{ Constant value } & 1.092 & 0.163 & - & $6.573^{* * *}$ & 0.736 & 0.286 & - & $4.095^{* * *}$ & 0.943 & 0.116 & - & $8.093^{* * *}$ \\
\hline \multirow{5}{*}{ Service quality } & Tangible & 0.022 & 0.093 & 0.026 & 1.993 & 0.220 & 0.109 & 0.227 & $4.997^{* * *}$ & 0.009 & 0.152 & 0.009 & 1.009 \\
\hline & Reliability & 0.244 & 0.103 & 0.231 & $4.993^{* * *}$ & 0.111 & 0.271 & 0.094 & 1.009 & 0.057 & 0.302 & 0.044 & 1.984 \\
\hline & Responsiveness & 0.024 & 0.028 & 0.033 & 0.668 & 0.061 & 0.095 & 0.075 & 0.886 & 0.078 & 0.931 & 0.088 & 2.094 \\
\hline & Confidence & 0.292 & 0.089 & 0.278 & $5.985^{* * *}$ & 0.228 & 0.386 & 0.194 & $3.971^{* *}$ & 0.283 & 0.042 & 0.220 & $3.547 * *$ \\
\hline & Empathy & 0.188 & 0.126 & 0.207 & $3.091 * *$ & 0.202 & 0.493 & 0.198 & $3.092 * *$ & 0.368 & 0.103 & 0.331 & $6.003^{* * *}$ \\
\hline \multicolumn{2}{|c|}{$\mathrm{R}^{2}$} & \multicolumn{4}{|c|}{0.413} & \multicolumn{4}{|c|}{0.416} & \multicolumn{4}{|c|}{0.351} \\
\hline \multicolumn{2}{|c|}{$\mathrm{F}$} & \multicolumn{4}{|c|}{$39.849^{* * *}$} & \multicolumn{4}{|c|}{$40.303^{* * *}$} & \multicolumn{4}{|c|}{$30.587 * * *$} \\
\hline
\end{tabular}

\subsection{Influence of Service Quality and Exercise Continuation Intention on Recommendation Intention}

The results of verifying the effect of service quality on recommendation intention through multiple regression analysis are shown in Table 7 . The effect of service quality on recommendation intention was statistically significant at a significance level of 0.001 , and the F-value of the regression model was $14.657 . R^{2}=0.206$ for the regression equation indicated that it had $20.6 \%$ explanatory power, and reliability, certainty, and empathy significantly affected recommendation intention.

Table 7. Influence of service quality on recommendation intention.

\begin{tabular}{|c|c|c|c|c|c|}
\hline & & \multicolumn{4}{|c|}{ Recommendation Intention } \\
\hline & & B & SE & $\beta$ & $t$ \\
\hline \multicolumn{2}{|c|}{ Constant value } & 2.392 & 0.209 & - & $7.284 * * *$ \\
\hline \multirow{7}{*}{ Service Quality } & Tangibility & 0.123 & & 0.132 & 2.001 \\
\hline & Reliability & 0.268 & & 0.232 & $4.028 * * *$ \\
\hline & Responsiveness & 0.074 & & 0.068 & 1.739 \\
\hline & Confidence & 0.293 & & 0.261 & $5.002 * * *$ \\
\hline & Empathy & 0.327 & & 0.373 & $5.913^{* * *}$ \\
\hline & & 0.206 & & \\
\hline & & $14.657 * * *$ & & \\
\hline
\end{tabular}

The results of verifying the effect of exercise continuity intention on recommendation intention with multiple regression analysis are shown in Table 8. The effect of exercise continuity intention on recommendation intention was statistically significant at the significance level of 0.001 , and the F-value of the regression model was $155.574 . R^{2}=0.352$ for the regression equation indicated that it had $35.2 \%$ explanatory power, and possibility and reinforcement significantly affected recommendation intention.

Table 8. Influence of exercise continuation intention on recommendation intention.

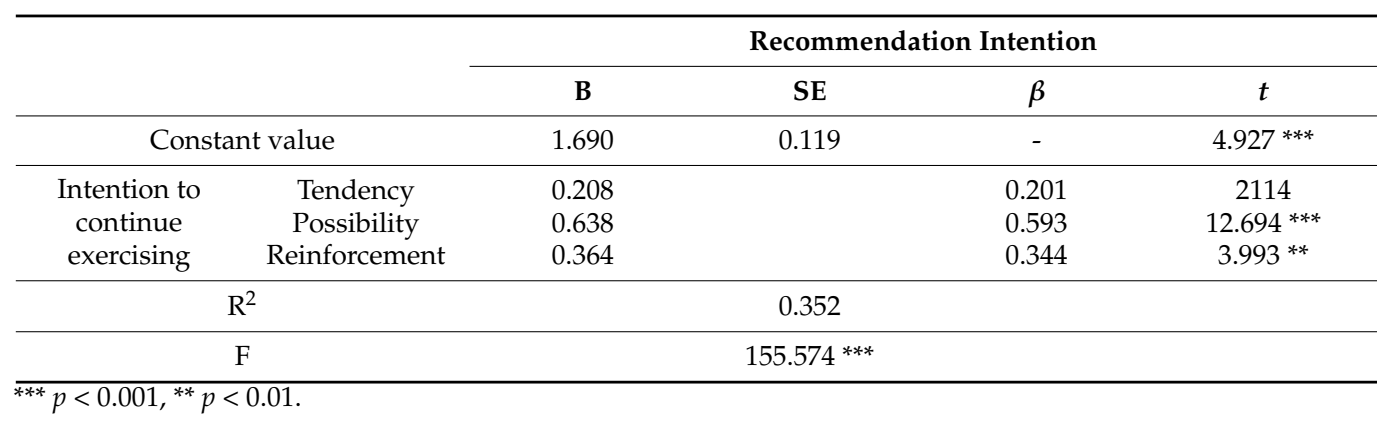




\section{Discussion}

Although Korea's Hapkido market has developed rapidly in terms of quantity and quality, it has not been able to find a solution to overcome the decline in Hapkido due to the recent economic recession in Korea and the fall in birth rate.

The quality of service for Hapkido refers to programs that enhance the value and function of education to provide training services. It is classified into tangibility, reliability, responsiveness, confidence, and empathy. Tangibility refers to external factors such as buildings, major facilities, and equipment evaluating Hapkido; reliability refers to the assessment of program batches and accuracy; and responsiveness involves problem solving, certainty, and comprehensive satisfaction, such as that related to facilities and equipment, program, and leadership qualities [22].

In this study, reliability, tangibility, confidence, and empathy were highly correlated with exercise continuation intention. Moreover, it was found that the overall satisfaction with the leader's qualities, and empathizing with the leader and the students, positively affected the intention to continue exercising. Therefore, the consistency and accuracy of the programs, a prepared environment for training, a comprehensive satisfaction with leaders and students, and empathy between them positively affect exercise continuation intention.

Studies on martial arts in South Korea, especially on the highly active Taekwondo gymnasiums, reported that the reliability of service quality affected reuse intention and the sustainability of the service [22,23]. Specifically, the reliability, certainty, and empathy of service quality positively affected continuation intention [24]. This indicates that improved reliability, conviction, and empathy of Hapkido services can lead to improvements in internal motivations, such as physical strength, athletic knowledge, belief, and confidence, that help individuals exercise daily.

That is, in the case of Hapkido in South Korea, trainees will be motivated to continue exercising if there are improvements in service quality, such as the implementation of promised training programs, trust in Hapkido leaders' expertise, and programs offering understanding and enjoyment. As a result, Hapkido trainees will have high exercise continuation intention and they will continue to exercise at regular intervals, resulting in positive effects on their health, physical condition, self-defense, physical improvement, and social relations.

Confidence and empathy factors relating to service quality are also closely related to possibility through external motivation factors [24], such as the programs, places, instruments, and equipment of exercise [25], which are important for athletic quality. This will help the students strengthen skills, such as time management.

The confidence and empathy factors relating to service quality are causally related to the reinforcement factor [24], which indicates the role of family, colleagues, and leaders in encouraging students to continue exercising regularly. This shows that if there is good leadership in Hapkido, trust between students and leaders, and smooth communication, the students will continue their current Hapkido training, regardless of location, thus ensuring vitality and enjoyment of life.

According to Greenwell et al. [26], the reliability, confidence, and empathy subfactors of quality of service have a positive impact on the trainees' recommendation intentions, consumer behavior, and future plans. This supports the results of the present study [27]. Thus, reinforcing reliability, confidence, and empathy in Hapkido services positively affects recommendation intention, which means improving satisfaction with service quality and benefits to others [28].

In the case of the causal relationship between students' exercise continuation and recommendation intentions, the possibility and reinforcement among the subfactors of the former significantly affect the latter. These results are supported by Kim et al. [29], who conducted a similar study on boxing and golf students and reported a positive effect on recommendation intention [30].

Therefore, for middle and high school students learning Hapkido in South Korea, the reinforcement of the ability to express satisfaction through exercise experience, improved 
physical strength, emotional stability, and trust between students and leader positively impacted their recommendation intention.

Like other martial arts, Hapkido can train the mind and body of individuals; in particular, it has a great and positive influence on teenagers' emotional and physical improvement and career decisions. To revitalize Hapkido in South Korea, improving the quality of service to smoothly operate Hapkido gymnasiums is, thus, very important.

\section{Limitations and Strengths}

This study has some limitations. First, it does not consider parents' opinions on external education for middle and high school students in the Korean context. Therefore, further studies may find additional ways to investigate the importance of motivation and revitalize the quality of service of the Hapkido market.

\section{Conclusions and Suggestions}

This study examined the effects of service quality factors as perceived by Hapkido trainees on their exercise continuation and recommendation intentions. The perceived service quality was measured by deriving the causal relationship between exercise continuation intention and recommendation intention, as shown in the preceding research, to reduce the dropout rate and strengthen competitiveness.

The findings of this study can help promote exercise and present recommendations to improve the satisfactory effect of quality of service for students. Specifically, the sophistication of facilities, programs, employees, and Hapkido facilities, which are relevant to the tangible factors relating to service quality, should be improved to deliver the promised services. In addition, immediate feedback should be provided to the requests of trainees, and a deep, trusting relationship should be built based on professional guidance. Regarding leaders, communication with students should be maintained based on their requirements.

This study suggests that various efforts should be made to increase the reliability, tangibility, confidence, and empathy of service quality, in order to increase the exercise continuation and recommendation intentions of the trainees of Hapkido gymnasiums.

Author Contributions: Writing-original draft preparation, J.K., T.-S.P.; data analysis, T.-S.P.; critical review of the contents, J.K., J.-S.K.; data collection and critical review of the manuscript, T.-S.P., J.-S.K.; supervision, T.-S.P., J.-S.K., J.K. All authors have read and agreed to the published version of the manuscript.

Funding: No source of funding.

Institutional Review Board Statement: Not applicable.

Informed Consent Statement: Not applicable.

Data Availability Statement: Data sharing not applicable.

Conflicts of Interest: The authors declare there are no conflict of interest.

\section{References}

1. Park, K.J.; Choi, J.K. A study on the process of forming career awareness of university students majoring in Hapkido through the university life and the Hapkido competition experience. J. Korean Alliance Martial Arts 2019, 21, 45-59.

2. Choi, B.H. Spatial diffusion and facility management of Hapkido gymnasiums in Germany. Korea Sport Res. 2007, 18, 321-328.

3. Lee, M.J.; Choi, D.M.; Kim, J.G. The effects of Hapkido training on sports confidence and brain waves in elementary school students. J. Korean Alliance Martial Arts 2019, 21, 201-212.

4. Choi, D.M.; Kim, J.G.; Lee, M.J. The effect of Hapkido training on personality development and leadership in adolescents. Korean J. Sports Sci. 2020, 29, 267-273. [CrossRef]

5. Ryan, R.M.; Deci, E.L. Intrinsic and extrinsic motivation from a self-determination theory perspective: Definitions, theory, practices, and future directions. Contemp. Educ. Psychol. Sci. 2020, 61, 1-11. [CrossRef]

6. Kalajas-Tilga, H.; Koka, A.; Hein, V.; Tilga, H.; Raudsepp, L. Motivational processes in physical education and objectively measured physical activity among adolescents. J. Sport Health Sci. 2020, 9, 462-471. [CrossRef] [PubMed]

7. Kim, E.Y.; Cho, S.J. Relation between recognition and actual practice of instructor according to education in Hapkido personality. Korean J. Sports Sci. 2017, 26, 969-982. [CrossRef] 
8. Lee, Y.J. The structural relationship among educational service quality on service value, trust and future behavior intention in Hapkido gymnasium. J. Korean Alliance Martial Arts 2020, 22, 177-190.

9. Lonial, S.; Menezes, D.; Tarim, M.; Tatoglu, E.Z. An evaluation of SERVQUAL and patient loyalty in an emerging country context. Total Qual. Manag. 2010, 21, 813-827. [CrossRef]

10. Anderson, E.W.; Sullivan, M.W. The antecedents and consequences of customer satisfaction for firm. Mark. Sci. 1993, 12, 125-143. [CrossRef]

11. Hay, J.M.; Hill, A.V. Service guarantee strength: The key to service quality. J. Oper. Manag. 2006, 24, 753-764.

12. Yi, Y.J. A critical review and future research directions on service quality, customer satisfaction, customer loyalty, and loyalty program. Korean Manag. Rev. 2016, 45, 1763-1787. [CrossRef]

13. Kang, S.H.; Yang, M.H. The effect of service quality of Taekwondo Dojang perceived by elementary students' parents on continuity intention: The serial multiple mediating effect of service satisfaction and Dojang loyalty. J. Korean Alliance Martial Arts 2019, 21, 61-76.

14. Cho, L.H. The impacts of the service quality of a Taekwondo gym on service reliability, the satisfaction with training and the intention to continue training. J. Korean Alliance Martial Arts 2020, 22, 159-175.

15. Hwang, J.H. Constructive relationships among service quality, visitation satisfaction, revisit intention, and recommendation intention of Taekwondo event. Korean J. Sports Sci. 2013, 22, 517-527.

16. Parasuramam, A.; Zeithaml, V.A.; Berry, L.L. SERVQUAL: A multiple-item sale for measuring consumer perception of service quality. J. Retail. 1988, 64, 12-40.

17. Corbin, C.B.; Lindsey, R. Concepts of Physical Fitness; Brown Communications: Dubuque, IA, USA, 1994.

18. Lee, K.O. Self-regulation, self-efficacy, and exercise adherence of physical education middle and high school students. J. Korean Soc. Stud. Phys. Educ. 2012, 17, 105-116.

19. Kim, H.R.; Park, S.M.; Jang, E.J. The relationship between environmental awareness and nature-friendly spending behavior of nature-friendly sports participants. Korean J. Sch. Phys. Educ. 2004, 14, 57-67.

20. Choi, J.L. The Structural Model for the Effects of Taekwondo Gym Service Quality Factors on the Parents' Satisfaction, Relationship Marketing, Continuance Intention and Recommendation Intention; Kyunghee University: Suwon, Korea, 2012; Unpublished doctor's dissertation.

21. Nunnally, J.C. Psychometric Theory; McGraw-Hill: New York, NY, USA, 1978.

22. Kim, J.T.; Kim, S.I.; Jung, Y.S. The relationship among service quality, customer satisfaction, and behavioral intentions of Taekwondo gymnasium. J. Korean Soc. Wellness 2011, 6, 63-73.

23. Hu, P.J.; Brown, S.A.; Thong, J.Y.; Chan, F.K.; Tam, K.Y. Determinants of service quality and continuance intention of online services: The case of eTax. J. Assoc. Inf. Sci. Tech. 2010, 60, 292-306. [CrossRef]

24. Corbin, C.B.; Lindsey, R.; Welk, G.J.; Corbin, W.R. Concepts of Physical Fitness and Wellness, 4th ed.; McGraw Hill Publishers: Dubuque, IA, USA, 2001.

25. Kim, S.Y.; Kim, B.S. An influence of a quality of personal training service on customer satisfaction, continued participation and oral transmitting intention. Korean J. Sport 2016, 14, 299-314.

26. Greenwell, T.C.; Fink, J.S.; Pastore, D.L. Assessing the influence of the physical sports facility on customer satisfaction within the context of the service experience. Sport Manag. Rev. 2002, 5, 129-148. [CrossRef]

27. Bolton, R.N.; Drew, J.H. A multistage model of customer' assessments of service quality and value. J. Consum. Res. 1991, 17, 375-384. [CrossRef]

28. Wang, Q.; Sun, Y.; Zhu, J.; Zhang, X. The impact of uncertain rewards on customers' recommendation intention in social networks. Internet Res. 2018, 28, 1029-1054. [CrossRef]

29. Kim, S.Y.; Cho, K.M.; Bae, J.S. The effect of customer satisfaction regarding boxing club service quality on exercise maintenance and recommendation intention. Korean Soc. Sports Sci. 2009, 28, 553-569. [CrossRef]

30. Lee, S.H.; Choo, J.H. The influence of subjective exercise experience in sports continuation and recommendation for others at college golf class. J. Sport Leis Stud. 2012, 47, 617-626. [CrossRef] 